\title{
Numerical Studies of Scrape-off Layer Connection Length in Wendelstein7-X
}

\author{
P. Sinha, ${ }^{*}$ H. Hölbe, T. S. Pedersen, S. Bozhenkov, and W7-X Team \\ Max-Planck-Institute for Plasma Physics, Wendelsteinstraße 1, 17491 Greifswald
}

(Dated: October 5, 2017)

\begin{abstract}
The distribution of particles and power flux to plasma-facing components play a critical role in the design of next-generation fusion devices. In particular, it is a challenge to bring down the peak heat fluxes onto the divertor to manageable levels (of order $5 \mathrm{MW} / \mathrm{m}^{2}$ ). A large value of connection length $\left(L_{c}\right)$ increases the role of cross-field transport and may lead to broader power deposition profile and lower peak heat fluxes. In low-shear stellarators like Wendelstein 7-X (W7-X) very long connection lengths, about one order of magnitude longer than those of equal-sized tokamaks, can be achieved. The flexibility of W7-X coil system allows variation of connection lengths significantly. This paper describes numerical studies that identify magnetic configurations in W7-X with particularly large and particularly small $L_{c}$; thus, the effects of $L_{c}$ on divertor operation can be effectively studied. It is found that a variation of a factor of 8 is possible, with average $L_{c}$ values as large as $426 \mathrm{~m}$ for a configuration developed here. It is also shown that $L_{c}$ itself, for a given magnetic configuration, varies strongly, potentially complicating an experimental analysis of its effects.
\end{abstract}

\section{INTRODUCTION}

The basic principle of an island divertor (ID) is that the plasma particle- and heat-fluxes are guided by field lines forming magnetic islands towards divertor targets placed away from the confined plasma in the core region, allowing for exhaust of the plasma particles as they neutralize [1]. Whereas in tokamaks, it is the rotational transform $\iota=1 / \mathrm{q}$ that makes the magnetic field lines wander towards the divertor, it is the internal rotational transform $\iota_{i}=r_{i} \iota^{\prime}$ inside the island that moves the field lines to the target plates in a stellarator, where $r_{i}$ the radial size of the island chain and $\iota^{\prime}$ the shear at the location of the island chain, and $L_{c}$ can be expressed as $L_{c}=2 \mathrm{R} / \mathrm{N} \iota_{i}$, where $\mathrm{R}$ the major radius and $\mathrm{N}$ the number of islands, ie. $L_{c}$ is very large in low shear stellarators like W7-X $[1,2]$.

Power exhaust is one of the major challenges in a future fusion power plant. In the scrape off-layer (SOL) region, the radial profiles are determined by competition between transport processes perpendicular and parallel to the magnetic field. SOL widths are of importance as they play a major role in characterizing the area of the divertor plate over which the outflowing plasma power is deposited. Because the parallel transport rate is orders of magnitude faster than the perpendicular transport rate, the SOL width is quite small. The heat load on the various components of the fusion device is a matter of great concern. A multitude of studies has been undertaken to determine the power fall-off length $\lambda_{q}$ $[9,10,11]$. In a multi-machine database for the H-mode $\lambda_{q}$ in JET, DIII-D, ASDEX Upgrade, C-Mod, NSTX and MAST, the regression inside the database showed that $\lambda_{q} \propto B_{\text {tor }}^{0.8} q_{95}^{1.1} P_{S O L}^{0.1} R_{\text {geo }}^{0}[7]$, for large aspect ratio $\mathrm{L} \approx \pi \mathrm{R} q_{S O L}$, thus exhibiting an approximately linear

\footnotetext{
* email: priyanjana.sinha@ipp.mpg.de
}

dependency with $L_{c}$. A heuristic model [8], in good agreement with the regression result, assumes that the magnetic gradient B and curvature drifts carry the charged particles across the last closed magnetic surface onto the open field lines in the SOL, and predicts linear proportionality between $L_{c}$ and $\lambda_{q}$. This supports the hypothesis that $L_{c}$ is a parameter that significantly affects the projected power decay length in future devices. $L_{c}$ also plays an important role in determining the temperature profile of the SOL plasma [3]. It has been observed in LHD that very long $L_{c}$ can reduce the heat load to the diverter while keeping the high performance of core plasma confinement intact [14]. Long connection lengths allows a significant temperature drop along field lines from hot upstream separatrix temperatures to reasonably low divertor target temperatures, and might play a role in accessing a high recycling regime [13] for these reasons, long connection lengths in the SOL are generally considered advantageous.

\section{COIL SYSTEMS AND THE NINE VACUUM REFERENCE CONFIGURATIONS}

W7-X is an optimized stellarator and uses 70 superconducting and 15 normal conducting coils for creating the magnetic field. The superconducting coils are responsible for generating the steady state main field to confine the plasma. Inside of the plasma vessel there are ten control coils to change the size of the islands and to sweep the islands poloidally and thereby modify the strike points of the plasma at the divertor. In addition, a set of five normal conducting trim coils increase the experimental flexibility, most notably by providing means to balance the asymmetric heat loads on the 10 divertor modules of W7-X. [4, 5, 6].

The W7-X has been optimised with respect to good MHD stability, improved neoclassical confinement, 
good confinement of fast particles, and low bootstrap current. Based on the optimization criteria, nine vacuum configurations have been defined as vacuum reference configurations (VRC), spanning a configurational space that allows studies of a number of interesting physical questions. Here, we investigate numerically how $L_{c}$ varies across these nine reference configurations, and, inspired by these results and some basic intuition, present a new magnetic configuration that has particularly high values of $L_{c}[6]$.

\section{NUMERICAL METHOD}

The method used for the numerical study of achievable $L_{c}$ for the $9 \mathrm{VRC}$ of $\mathrm{W} 7-\mathrm{X}$ is field line tracing. A focus has been given on $L_{c}$ at the positions where the divertor is in direct contact with relatively hot and dense SOLplasma obtained using field line diffusion, also referred to as "wetted area" or "strike line" , as it receives the direct heat load from the plasma and is relevant from the point of view of the safety of the divertor. The magnetic field is computed using the Biot-Savart law and then, after finding the last closed flux surface (LCFS) for the magnetic configuration being studied, starting points are placed on it. The starting points are traced along the magnetic field line, with small random steps perpendicular to the field included to simulate perpendicular diffusion [13]. The tracing process continues until the field line hits any in-vessel material component, such as divertors, baffles, heat shields etc. The parameters to describe the perpendicular diffusion process are perpendicular diffusion coefficient $D_{\perp}$, mean free path $\lambda$, and velocity $\mathrm{v}$. They can be adjusted to change the effective perpendicular diffusion rate. The perpendicular step are performed after a random length $\mathrm{x}$ with the distribution $p_{x}=1 / \lambda$ $\exp (\mathrm{x} / \lambda)$. For these studies, we have chosen the value of perpendicular diffusion (D) to be $1 \mathrm{~m}^{2} / \mathrm{s}$ based on the previous experiments in W7-AS. The parallel diffusion velocity in the code

$$
<v>=\sqrt{\frac{k_{B}\left(T_{e}+T_{i}\right)}{m_{e}+m_{i}}}
$$

corresponds to a temperature at the edge of approximately $1 \mathrm{keV}$ [4] Although the edge temperature is unrealistically high, it is convenient since it gives localized wetted area and therefore better statistics for the region of the divertor with highest heat loads. The position and the number of strike lines varies for each of the 9 VRC. Points on the divertor found this way are points where plasma heat load is predicted to be present, since the plasma can diffuse across the magnetic field in a way similar to the random perpendicular steps taken. The magnetic field line going through this loaded divertor-surface point is then followed back in the direction from which it came, this

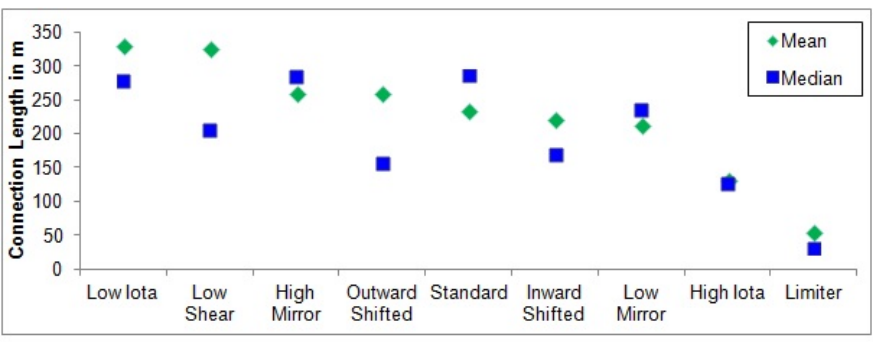

FIG. 1. Graph of Mean and Median of Connection Length vs the $9 \mathrm{VRC}$

time without taking any perpendicular steps, until the field line reaches a material surface again (typically the surface of a different divertor). Thus, the connection length of plasma-filled SOL field lines can be determined.

\section{RESULTS}

The average value of $L_{c}$ was calculated and compared for all 9 VRC. Limiter is a special configuration where the LCFS connects directly to the target plates, whereas for the other 8 configurations, the magnetic island chain at the edge intersects the target plates. Fig.1 implies that Low Iota (with an island chain with $\mathrm{m}=6$ ) has the highest value of mean $L_{c}(331 \mathrm{~m})$, whereas the Limiter configuration without islands at the edge, has the lowest value of mean $L_{c}(55 \mathrm{~m})$. This demonstrates that the islands in the ID concept are in fact responsible for the long connection length obtained in $\mathrm{W} 7-\mathrm{X}$. The wide variation in the range of connection length illustrates the flexibility of the W7-X coils system.

There is a wide variation of $L_{c}$ values in the wetted area, raising the concern that the mean values alone do not suffice to describe the structure or width of the wetted area. We illustrate this in Fig.2, where we plot strike line patterns for the Limiter configuration on the divertor with the color at each point indicating $L_{c}$ at that point. One sees that connection lengths very small and very large (up to $2000 \mathrm{~m}$ ) are present. This feature of large variance in connection length in a magnetic configuration has also been observed in other stellarator and heliotron devices like LHD $[15,16]$. Two strike lines are visible on the divertor plate, one on the horizontal divertor plate elongated toroidally and the other on the vertical divertor plate (Fig.2). It is observed that the large densities of points are in orange or yellow color with $L_{c}$ of $30-40 \mathrm{~m}$ i.e the field line travels approx. 1 turn around the machine before hitting a component. This brings down the overall mean value of connection length to a low value despite there being some very long connection lengths present as well. 


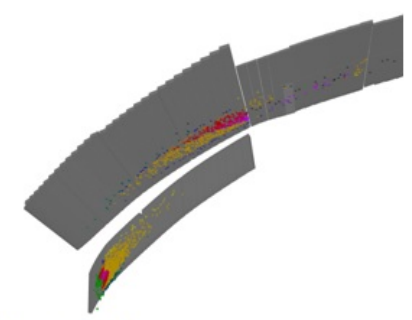

Color Scale of $L_{\varepsilon}$ from 0 to $2000 \mathrm{~m}$ :

FIG. 2. Footprints of field lines on divertor targets for Limiter Configuration using field-line diffusion wherein color at each point corresponds to the $L_{c}$ at that point. $\left(\mathrm{D}=1 \mathrm{~m}^{2} / \mathrm{s}\right.$ and $\left.\mathrm{V}=0.5 \cdot 10^{6} \mathrm{~m} / \mathrm{s}\right)$

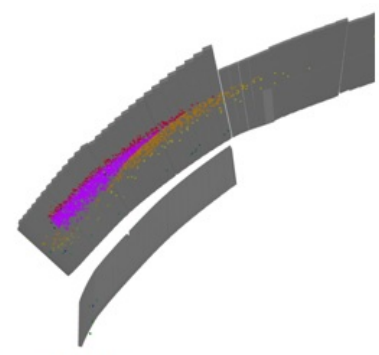

Color Scale of $L_{c}$ from 0 to $2000 \mathrm{~m}$ :

FIG. 3. Footprints of field lines on divertor targets for Low Iota Configuration using field-line diffusion wherein color at each point corresponds to the $L_{c}$ at that point $\left(\mathrm{D}=1 \mathrm{~m}^{2} / \mathrm{s}\right.$ and $\left.\mathrm{V}=0.5 \cdot 10^{6} \mathrm{~m} / \mathrm{s}\right)$

In the Low Iota configuration, only one strike line is observed on the horizontal divertor plate with a long toroidal extension (Fig.3). The strike points contain yellow $(20-40 \mathrm{~m})$, orange $(40-80 \mathrm{~m})$, red $(80-190 \mathrm{~m})$ and a large proportion of points in $\operatorname{pink}(>190 \mathrm{~m})$ color i.e field line travels for more than 5 turns around the machine before hitting a component, therefore the final average $L_{c}$ is quite large. It is found that Low Iota and Low Shear have the largest values of mean $L_{c}, 331 \mathrm{~m}$ and $326 \mathrm{~m}$ respectively. We show in the following that a new configuration that is a combination of of Low Shear and Low Iota has even higher values of $L_{c}$.

The New Configuration is a configuration that has particularly low shear in combination with a $5 / 6$ (low iota) island chain. Its modular coil currents are similar to those of the Low Shear configuration, and its planar coil currents obtained by adding the currents in the Low Shear and the Low Iota configurations. The mean $L_{c}$ of this configuration is $426 \mathrm{~m}$ (Fig.4), significantly higher than the mean $L_{c}$ of any of the $9 \mathrm{VRC}$.

The Poincaré plot [4] for this New Configuration (Fig.6) features islands just touching the divertor plate

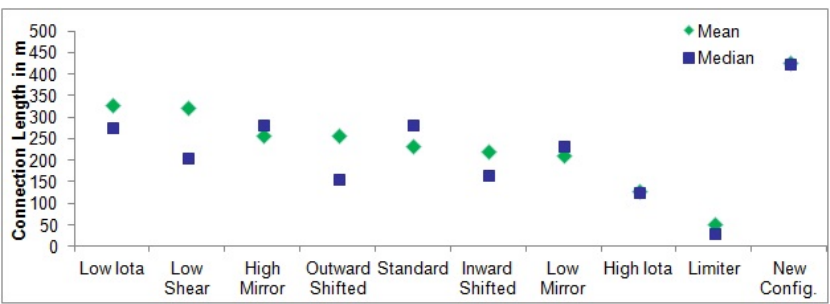

FIG. 4. Graph of Mean and Median of Connection Length vs the $9 \mathrm{VRC}$ and the New Configuration showing the flexibility of W7-X coil system in terms of attainable connection length

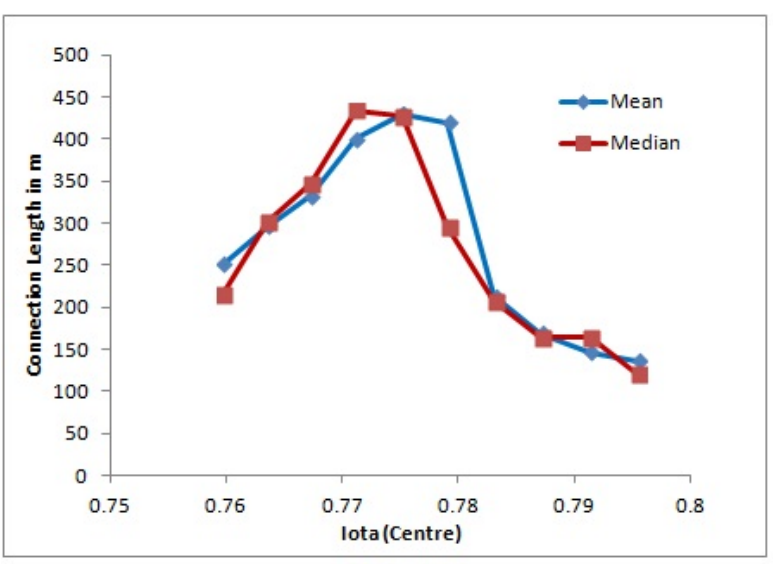

FIG. 5. The Iota Scan using iteration for the New Configuration depicting small variation in planar coil current altering the $L_{c}$ significantly

at toroidal angle $\phi=0$ plane; this presumably contributes to the high $L_{c}$ obtained in this configuration. This interpretation is supported by an iota scan around this configuration, which was carried out by varying the planar coil current (Fig.5). Varying iota shifts the island chain radially in or out, and one sees that the long connection lengths are rather narrowly distributed for the iota values (island locations) very close to those of the New Configuration.

The $L_{c}$ is evaluated and the Poincaré plot is noted for the altered configurations. It was noticed that the average $L_{c}$ values dipped considerably with slight modification in planar coil current in the New Configuration showing the significant impact of small changes in planar coil current on the position of island w.r.t divertor which directly impacts the connection length achievable.

\section{CONCLUSION}

The present numerical study on the SOL connection length $L_{c}$ in W7-X with a focus on $L_{c}$ at the strike line position, demonstrates that $L_{c}$ varies from approx- 


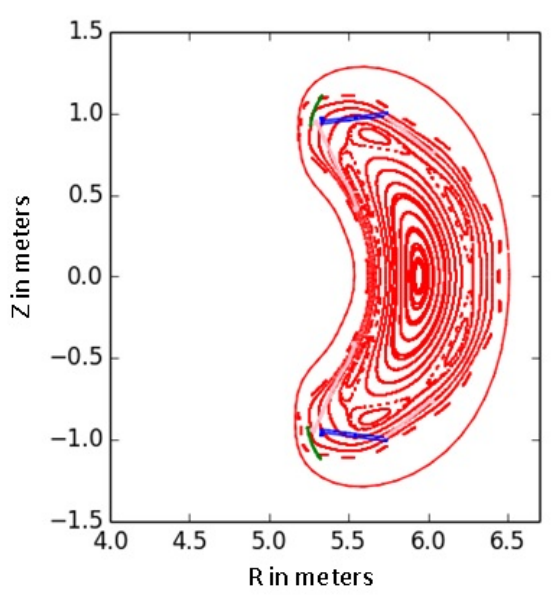

FIG. 6. The Poincaré plot for New Configuration with island just touching the divertor(in blue) imately $55 \mathrm{~m}$ to approximately $330 \mathrm{~m}$ in the 9 vacuum reference configurations. Taking further advantage of the flexibility of the W7-X coil system, a new configuration was identified with a particularly high value of average $L_{c}$ of $426 \mathrm{~m}$. This span of almost an order of magnitude in $L_{c}$ should allow a determination of the importance of $L_{c}$ for divertor operation in general and for the value of $\lambda_{q}$ in particular, although the large spread in $L_{c}$ values for a given configuration could complicate such an analysis.

\section{ACKNOWLEDGEMENTS}

This work has been carried out within the framework of the EUROfusion Consortium and has recieved funding from the Euratom research and training programme 2014-2018 under grant agreement No 633053. The views and opinions expressed herein do not necessarily reflect those of the European Commission.
[1] Feng, Y., et al. Comparative divertor-transport study for helical devices. Nuclear Fusion 49.9 (2009): 095002.

[2] Feng, Y., et al. Physics of island divertors as highlighted by the example of $W^{7}$-AS. Nuclear fusion 46.8 (2006): 807.

[3] Sardei, F., et al. Island divertor: Concepts and status of experimental and modelling results. No. IAEA-CSP-8/C. W7-AS Team, 2001.

[4] Hölbe, Hauke. Control of the magnetic topolgy and plasma exhaust in the edge region of Wendelstein 7-X: A numerical study. (2016).

[5] Rummel, Thomas, et al. The Wendelstein 7-X trim coil system. IEEE Transactions on Applied Superconductivity 24.3 (2014): 1-4.

[6] Andreeva, T., J. Kisslinger, and H. Wobig. Characteristics of main configurations of Wendelstein 7-x. Problems of Atomic Science and Technology. Series: Plasma Physics (2002): 45-47.

[7] Eich, Thomas, et al. Scaling of the tokamak near the scrape-off layer H-mode power width and implications for ITER. Nuclear fusion 53.9 (2013): 093031.

[8] Goldston, R. J. Heuristic drift-based model of the power scrape-off width in low-gas-puff $\mathrm{H}$-mode tokamaks. Nuclear Fusion 52.1 (2011): 013009.

[9] Connor, J. W., et al. Comparison of theoretical models for scrape-off layer widths with data from COMPASS-D,
JET and Alcator C-Mod. Nuclear fusion 39.2 (1999): 169.

[10] Thornton, A. J., A. Kirk, and MAST Team. Scaling of the scrape-off layer width during inter-ELM $H$ modes on MAST as measured by infrared thermography. Plasma Physics and Controlled Fusion 56.5 (2014): 055008.

[11] Sieglin, B., et al. Investigation of scrape-off layer and divertor heat transport in ASDEX Upgrade L-mode. Plasma Physics and Controlled Fusion 58.5 (2016): 055015.

[12] Stangeby, Peter C. The plasma boundary of magnetic fusion devices. . Vol. 224. Bristol: Institute of Physics Publishing, 2000.

[13] S.A. Bozhenkov et. al. Service oriented architecture for scientific analysis at $W 7-X$. An example of a field line tracer. Fusion Engineering and Design, 88(11), 2013

[14] Watanabe, T., et al. Magnetic field structure and confinement of energetic particles in the LHD. Nuclear fusion 46.2 (2006): 291

[15] Suzuki, Y., et al. Impact of magnetic topology on radial electric field profile in the scrape-off layer of the Large Helical Device. Nuclear Fusion 56.9 (2016): 092002.

[16] Watanabe, K. Y., et al. Characteristics of MHD Equilibrium and Related Issues on LHD. Fusion Science and Technology 58.1 (2010): 160-175 